\title{
CRISPR/Cas9-mediated knockout of an oil palm defense-related gene to the pathogenic fungus Ganoderma boninense
}

\author{
Asmini Budiani ${ }^{1}$, Imam Bagus Nugroho ${ }^{1}$, Dini Astika Sari ${ }^{1}$, Inez Palupi ${ }^{2}$, and Riza Arief Putranto ${ }^{1,{ }^{*}}$ \\ ${ }^{1}$ Indonesian Research Institute for Biotechnology and Bioindustry, Jalan Taman Kencana No. 1, Bogor, Jawa Barat 16128, Indonesia \\ ${ }^{2}$ Agrotechnology Department, Faculty of Agriculture, Universitas Jenderal Soedirman, Jalan dr. Suparno PO BOX 125 Purwokerto, Jawa \\ Tengah 53123, Indonesia \\ *Corresponding author: rizaputranto@iribb.org
}

SUBMITTED 1 July 2019 REVISED 23 August 2019 ACCEPTED 11 December 2019

\begin{abstract}
Oil palm plantation in Indonesia is significantly affected by basal stem rot disease caused by the pathogenic fungus Ganoderma boninense. Tolerant oil palm cultivars toward G. boninense have been developed through a breeding program accelerated by the implementation of the CRISPR/Cas9 technology. This study was conducted to perform a gene knockout $(\mathrm{KO})$ of oil palm that confers a putative defense-related trait toward G. boninense. A plasmid pCRISPR_EMLP containing modules, i.e., 35S-CaMV-promoter-driven CRISPR/Cas9, U6-promoter-driven sgRNA to the target EgEMLP gene (EL695076), and hygromycin resistance gene as the selectable marker, was established for Agrobacterium-mediated delivery into oil palm calli (OPC). The transformed OPCs were regenerated and screened in DF (de Fossard) media containing hygromycin. The working concentration of hygromycin was successfully optimized for selection at $20 \mathrm{ppm}$. Through PCR-based selection using HYG primers, we succeeded in discerning positive transformed OPC clones. The sequenced PCR products of genomic DNA as the template amplified using EMLP1 primers showed a point mutation, causing a frameshift in the edited EgEMLP and premature stop codon. Furthermore, in silico modeling demonstrated that the mutation resulted in a change in the $\mathrm{C}$-terminal region, affecting the tertiary protein structure. Moreover, electrophoresis analysis of PCR products of cDNA as the template from transformed OPC clones showed several samples with faint or undetected bands. This indicated that the CRISPR/Cas9 module induced a mutation that could destabilize the transcribed mRNA, e.g., premature degradation. Altogether, this study has successfully implemented CRISPR/Cas9 gene editing in oil palm in a model gene that is responsible for putative defense-related traits toward the pathogenic fungus $G$. boninense.
\end{abstract}

KEYWORDS gene editing; plant-microbe interaction; Ganoderma boninense; defense trait; SNP

\section{Introduction}

Basal stem rot (BSR) disease that affects the oil palm is caused by the pathogenic fungus Ganoderma boninense (Ho and Tan 2015). This disease affects a wide area of oil palm plantation in Indonesia, causing devastating economic losses amounting to 500 million USD every year (Ommelna et al. 2012; Hushiarian et al. 2013). Recently, several efforts have been made to overcome the BSR disease. However, reported infections, casualties, and losses still appear to increase every year.

Currently, the development of tolerant oil palm cultivars against $G$. boninense is considered as the best solution. An engineered tolerant cultivar can be established using a breeding program from parent stocks with known traits resisting G. boninense. However, this traditional program is tedious and requires a long time to generate an improved trait.

An accelerated breeding program can be achieved by the implementation of cutting-edge technology, e.g., CRISPR/Cas9-mediated gene editing (Jaganathan et al. 2018). It has been reported that the CRISPR/Cas9 system naturally occurs in bacteria, which functions as an immunity element against virus (phage) invasion by incising the foreign DNA fragments (Horvath and Barrangou 2010). This system was repurposed and engineered to break the DNA target at a specific site using programmable guide RNA (sgRNA) (Jinek et al. 2012). The programed sgRNAs are generally synthetically built adjoining the Cas9 module in a suitable expression plasmid. When expressed, the Cas9 enzyme acts as a homing endonuclease directed by the sgRNA, which can hybridize to a particular site within the targeted DNA (Mali et al. 2013). Cas9 induces double-strand breaks (Shen et al. 2017), which are later repaired by the intracellular machinery. Edits are introduced in this manner in the form of point mutation, insertion, and deletion through the nonhomologous endjoining (NHEJ) pathway (Chiruvella et al. 2013) or by 
gene replacement through homology-directed recombination (Hahn et al. 2018). Therefore, the trait of interest can be generated using CRISPR/Cas9 using advantageous cisgenesis (Hou et al. 2014), avoiding the introduction of any transgenes (Telem et al. 2013).

In this study, we aimed at improving a trait of oil palm against infection with $G$. boninense. This was achieved by conducting a gene knockout (KO) experiment on the defense-related gene against the pathogenic fungus. The target gene was determined from another study that had demonstrated that the expression of EgEMLP was elevated during infection with $G$. boninense in oil palm. We hypothesized that EgEMLP is a marker gene that, if knocked out, will alter the oil palm trait, preferably increasing its tolerance toward $G$. boninense. In this study, we succeeded in knocking out EgEMLP by demonstrating several post-editing effects at the genomic (DNA) and transcriptomic (RNA) levels and through protein modeling. Further study is important to examine the growth of the edited oil palm clones under $G$. boninense infection in the field.

\section{Materials and Methods}

\subsection{CRISPR/Cas9 editing module design}

CRISPR/Cas9 modules were designed according to an earlier pipeline study (Budiani et al. 2018). The editing module was constructed to the target EgEMLP gene (EL695076). It was selected as described in a previous study that demonstrated its elevated expression in oil palm leaves upon G. boninense infection (Tan et al. 2013). We hypothesized that the CRISPR/Cas9-mediated knockout on EgEMLP would repress its expression, thereby altering the trait of the edited oil palm.

Optimum designed sgRNAs were assessed to examine the on-target specificity toward the target gene and the offtarget probability toward nontarget sites. One optimum sgRNA was selected for synthesis and assembly into the expression plasmid (Figure 1) using the services of SigmaAldrich.

\subsection{Agrobacterium-mediated transformation, calli re- generation, and screening}

The transformation was delivered using Agrobacteriummediated protocols as optimized in previous research (Budiani et al. 2019). The transformed oil palm calli (OPC) were regenerated in de Fossard (DF) media supplemented

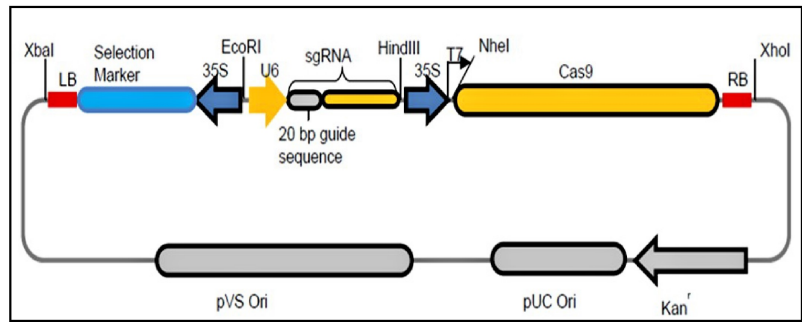

FIGURE 1 CRISPR_EMPLP construct containing Cas9, sgRNA for EgEMLP, and hygromycin selectable marker expression modules.

with hygromycin. Optimization of hygromycin for the selective marker was conducted using working concentrations of 10, 20, and $30 \mathrm{ppm}$. The working concentration for supplementation was considered to be lethal if it can induce calli browning. The optimal working concentration was deduced as the concentration at one level lower below the lethal concentration. Screenings were conducted to select the population of calli, which can survive under the supplementation of hygromycin at the optimum working concentration. They were then subjected to further screening.

\subsection{DNA and RNA isolation, cDNA synthesis, and elec- trophoresis}

All samples were ground using mortar and pestle in liquid nitrogen. Genomic DNA from calli was extracted using the Genomic DNA Mini Kit Plant (Geneaid) according to the manufacturer's instruction. Total RNA was extracted using the RNAEasy Plant Mini Kit (Qiagen). First-strand cDNA synthesis was conducted using the iScript cDNA Synthesis Kit (BIO-RAD). The electrophoresis was performed in an agarose gel ( $0.5 \%$ for RNA or $0.8 \%$ for DNA) in $1 \times$ TBE buffer in DEPC-treated or nuclease-free water.

\subsection{PCR-based selection, sequencing, and sequence analysis}

PCR experiments were conducted to screen for positive transformed OPC clones using $H Y G$ and EMLP1 primers (Table 1). The HYG primers were designed to amplify the hygromycin resistance gene. Meanwhile, the EMLP1 primers were designed to flank the regions targeted by the sgRNA where the Cas9 protein will cut and cause a double-strand break.

The PCR experiments using EMLP1 were conducted using genomic DNA and cDNA as templates to observe

TABLE 1 Oligonucleotides used in this study.

\begin{tabular}{lll}
\hline Names & Forward/Reverse & Sequences \\
\hline EMLP1 & Forward & AGAGCGTTTGGCTGAAGGT \\
& Reverse & AGAACTGCGCGCTCTAAGAC \\
HYG & Forward & ACTATCGGCGAGTACTTCTACAC \\
& Reverse & GTATCACTGGCAAACTGTGATG \\
Target site & - & TGAAGGGGTCGATATCGACGAGG*
\end{tabular}

*Bold nucleotides indicate PAM (Proto Adjacent Motif) sequence 
the changes introduced by the CRISPR/Cas9 modules at the genomic and transcriptomic levels. The reactions were performed using KAPPA 2G Fast Mix according to the manufacturer instructions in programmed cycles (35 cycles), i.e., initial denaturation at $95^{\circ} \mathrm{C}$ for 3 mins, denaturation at $95^{\circ} \mathrm{C}$ for $15 \mathrm{~s}$, annealing at $55^{\circ} \mathrm{C}$ for $15 \mathrm{~s}$, extension at $72^{\circ} \mathrm{C}$ for $15 \mathrm{~s}$, and a final extension at $72^{\circ} \mathrm{C}$ for 7 mins. The PCR products obtained from amplification using genomic DNA as the template was sequenced using Sanger Sequencing at 1st Base, Selangor, Malaysia. Sequence analyses were conducted in Geneious Prime suite (Biomatters, Ltd.).

\subsection{In silico modeling of partial EgEMLP protein struc- ture}

The amino acid sequences of EgEMLP exon of wild-type (nonedited) and transformed (edited) were translated using a standard genetic code in frame with the reference gene in Geneious Prime suite (Biomatters, Ltd.). The amino acid sequences were submitted to the I-TASSER server (Yang 2007) to yield the protein structure model. Idealized models were retrieved separately and saved as. PDB file. Both models were superimposed in the PyMOL suite (Schrödinger LLC). A descriptive analysis was conducted on the superimposed models to evaluate the differences between edited and nonedited models.

\section{Results and Discussion}

\subsection{Transformation and optimization of hygromycin working concentration for calli screening}

Screened calli were further selected by PCR-based selection using HYG and EMLP1 primers to confirm the transformations of the pCRISPR_EMLP construct. The electrophoresis profile obtained by PCR-based screening using HYG primers demonstrated successful OPC transformation using the pCRISPR_EMLP construct (Figure 3, upper panel). Meanwhile, the application of 10 ppm hygromycin was considered to produce false-positive results that allowed the growth of most of the calli. Therefore, the optimum working concentration of hygromycin was determined as 20 ppm, even if it could induce calli browning after 4 and 8 weeks of culture (Figure $2 \mathrm{D}$ and E). In this case, the results demonstrated that a gradually lowered hygromycin working concentration should be used during calli subcultures. Further screening procedures should also be implemented, e.g., PCR-based selection.

\subsection{PCR-based screening of CRISPR/Cas9-edited calli}

Screened calli were further selected by PCR-based selection using HYG and EMLP1 primers to confirm the transformations of the pCRISPR_EMLP construct. The electrophoresis profile obtained by PCR-based screening using HYG primers demonstrated successful OPC transformation using the pCRISPR_EMLP construct (Figure 3, upper panel). All the examined calli harbored the hygromycin resistance gene with an expected amplicon size of 700

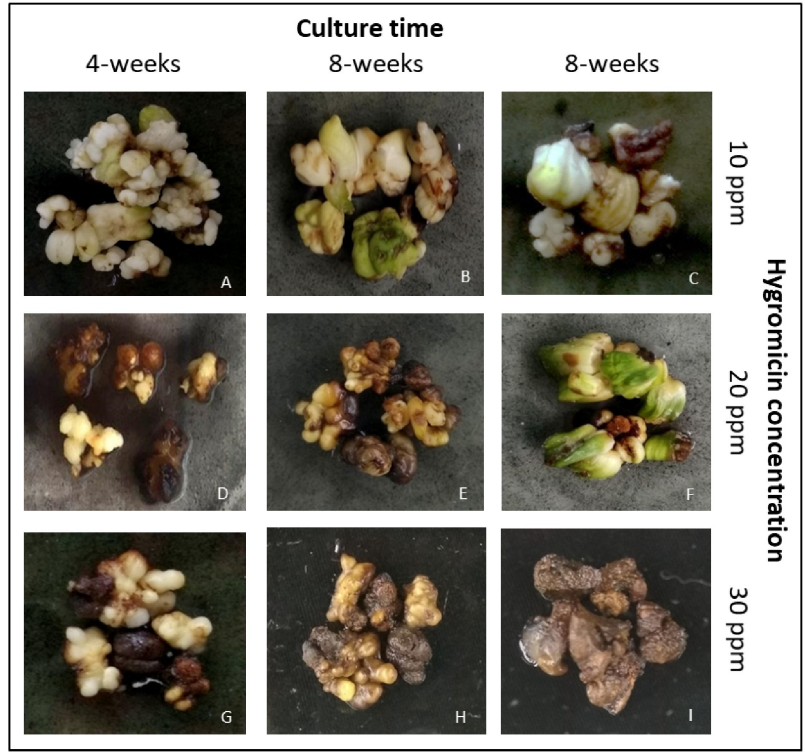

FIGURE 2 Calli selection in DF media supplemented with hygromicin. All calli were grown with $250 \mathrm{ppm}$ cefotaxime to reduce Agrobacterium tumefaciens over growth. Based on this result, the optimum hygromycin concentration for calli selection was deduced at $20 \mathrm{ppm}$, as higher concentration (30 ppm) will induce calli death (browning) while lower concentration at $10 \mathrm{ppm}$ will produce nontransformed (escapee) calli thus allowing for false positives.

bp, which conferred the ability to grow in media supplemented with hygromycin. PCR-based experiments were also established using $E M L P 1$ primers to examine the editing events at the genomic level. The PCR was able to produce the expected distinct amplicon at the size of $300 \mathrm{bp}$ using the genomic DNA as the template (Figure 3A.). Further examinations were conducted to confirm the editing events using Sanger sequencing of these amplicons.

PCR experiments were also conducted using EMLP1 primers on cDNA templates to examine whether the editing events can be discerned at the transcriptomic (mRNA) level. The electrophoresis profile revealed that some calli produced faint or none of the expected bands (-200 bp) (Figure 3B.), which indicated that editing events could be discerned at the transcriptomic level.

In this study, we established a procedure to screen OPC by the CRISPR/Cas9-mediated editing technology using PCR and electrophoresis on the cDNA template. We succeeded in demonstrating the CRISPR/Cas9-induced knockout (KO), which can later change the level of transcribed mRNA as shown by the faint or none of the expected bands. Therefore, the edited and nonedited calli obtained by the CRISPR/Cas9-mediated KO can be differentiated using routine PCR.

\subsection{Sequence analysis of CRISPR/Cas9-edited EgEMLP gene}

Sequence analysis of the PCR amplicons produced using the genomic DNA template was conducted using alignment against an identical genomic DNA template from wild-type sequences. This analysis demonstrated that one of the edited calli harbored a mutation (Figure 4) located at 

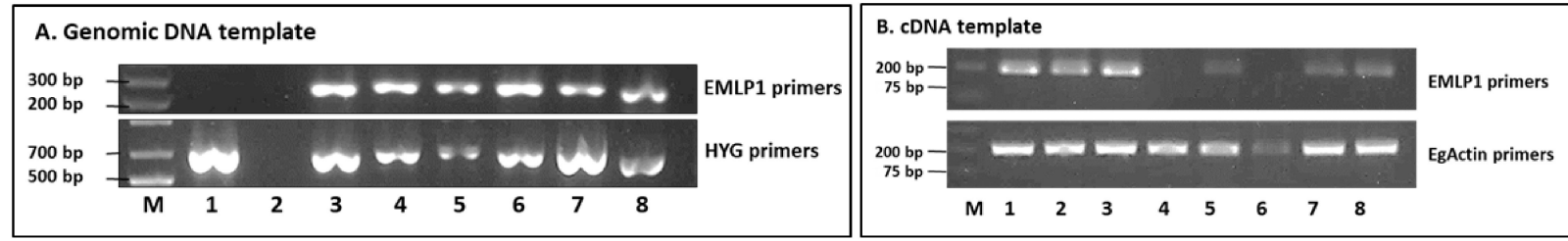

FIGURE 3 Electrophoresis profiles of PCR-based amplifications using specific primers. A. Genomic DNA template (M: 1kb+ DNA Marker, 1: hygromycin positive control, 2: negative control (nuclease-free water), and 3-8: transformed calli) and cDNA templates. B. cDNA template (M: 1kb+ DNA Marker, 1 - 8: transformed calli).

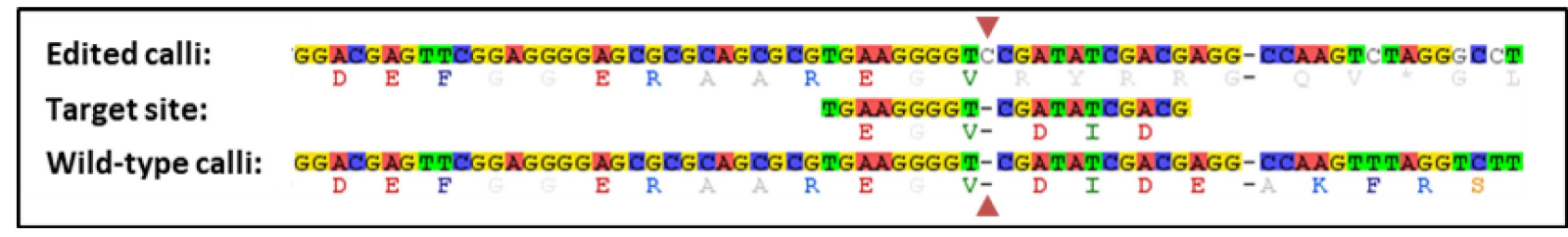

FIGURE 4 The pairwise sequence alignment of the edited calli against wild-type EgEMLP sequence produced in Geneious Prime suite. Highlighted nucleotide by red triangles showed where the change made by CRISPR/Cas9 creating SNP (single nucleotide polymorphism) and changing the translated amino acid sequence.

the CRISPR/Cas9 target site for EgEMLP. This indicated that the mutation occurred as a base insertion due to internal DNA repair after the double-strand break-induced by CRISPR/Cas9. This mutation induced a frameshift on the translated amino acid sequence, causing a change in the translated protein starting at the point mutation onward. It also introduced a premature stop codon in the edited sequence.

\subsection{Homology-based modeling for CRISPR/Cas9- edited EgEMLP protein}

In silico modeling was implemented to determine the effect of the mutation on the protein structure. As shown in Figure 5, the mutation distinctively changed the tertiary structure of EgEMLP protein based on the superimposed structures between the edited and wild-type proteins. The mutation resulted in the premature stop codon and deleted nine amino acid residues at the C-terminal region of EgEMLP protein. This change, in turn, affected

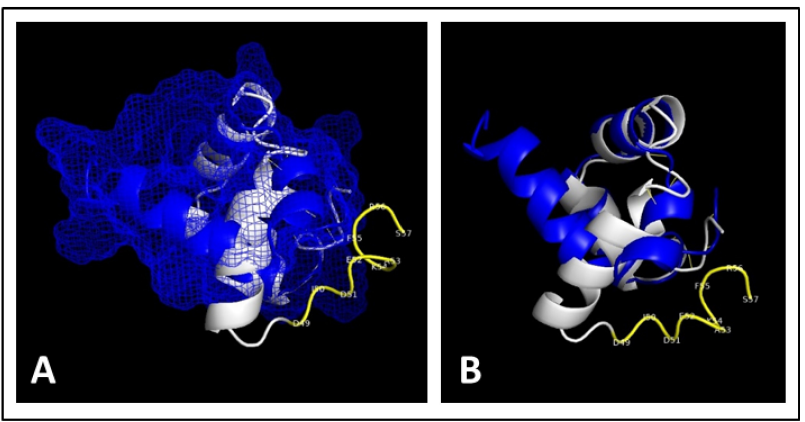

FIGURE 5 Superimposed models of wild-type (white) and edited (blue) tertiary structures of EgEMLP protein. A. Modelled structures of EgEMLP with mesh drawing. B. Tilted similar models visualised showing structurally unaligned alpha-helix between WT and edited proteins. Unstructured domain highlighted by yellow color was the deleted domain of the edited $E g E M L P$ due to a premature stop codon. the adjacent alpha-helix structure, indicating disruption of the tertiary structure, which may abolish the protein function.

Altogether, this study was successful in implementing the CRISPR/Cas9 editing technology to the engineering of OPC. Moreover, the optimum working concentration of hygromycin used in calli transformation screening was established, and it was deduced that a gradually lowered hygromycin working concentration could avoid falsepositive results. Furthermore, successful implementation of CRISPR/Cas9-mediated editing was demonstrated using results evidenced at multiple levels ranging from genomic, transcriptomic, and protein modeling. This study could open up the possibility of engineering crops, particularly with long growth periods, e.g., the oil palm, according to various applications, through the implementation of the cutting-edge marker-assisted molecular plant breeding program.

\section{Conclusions}

In this study, we had successfully implemented the CRISPR/Cas9 system to conduct a gene knockout in the OPC. We also successfully demonstrated the editing events using multiple pieces of evidence at the genomic and transcriptomic levels. Protein models of the edited and nonedited gene were also generated to predict the structural change that may cause disruption in its function.

\section{Acknowledgments}

The authors thank Marini, Elizabeth Monica Sitorus, and Nazhirotul Ilmiyah for the assistance. This research was funded by the BPDPKS (Indonesian Oil Palm Research Funding Agency). 


\section{Authors' contributions}

$\mathrm{AB}$ and RAP designed the research. RAP designed the pCRISPR_EMLP expression cassettes and generated the in silico EgEMLP model protein. DAS and IP conducted the OPC cultures and screening in antibiotic supplemented plates. IBN, DAS, and IP conducted the isolation of genomic DNA, PCR, sequencing, and subsequent analyzes. IBN isolated the RNA, conducted cDNA synthesis, PCR, electrophoresis, sequence analysis, and data compiling and analysis and wrote the manuscript. All authors read and approved the final version of the manuscript.

\section{Competing interests}

The authors declare no competing interest.

\section{References}

Budiani A, Nugroho IB, Minarsih H, Riyadi I. 2019. Regeneration of oil palm plantlets introduced by P5CS gene using Agrobacterium-mediated transformation. E-Journal Menara Perkebunan. 87(2):123130. doi:10.22302/iribb.jur.mp.v87i2.336.

Budiani A, Putranto RA, Riyadi I, Minarsih H, Faizah R. 2018. Transformation of oil palm calli using CRISPR/Cas9 System: toward genome editing of oil palm. In: IOP Conf. Series: Earth and Environmental Science (2018) 183, 012003, Jakarta, Indonesia. p. 012003. doi:10.1088/1755-1315/183/1/012003.

Chiruvella KK, Liang Z, Wilson TE. 2013. Repair of double-strand breaks by end joining. Cold Spring Harbor Perspect Biol. 5(5):a012757. doi:10.1101/cshperspect.a012757.

Hahn F, Eisenhut M, Mantegazza O, Weber APM. 2018. Homology-directed repair of a defective glabrous gene in Arabidopsis with Cas9-based gene targeting. Front Plant Sci. 9:424. doi:10.3389/fpls.2018.00424.

Ho CL, Tan YC. 2015. Molecular defense response of oil palm to Ganoderma infection. Phytochemistry. 114:168-177. doi:10.1016/j.phytochem.2014.10.016.

Horvath P, Barrangou R. 2010. CRISPR/Cas, the immune system of bacteria and archaea. Science. 327(5962):167-170. doi:10.1126/science.1179555.

Hou H, Atlihan N, Lu ZX. 2014. New biotechnology enhances the application of cisgenesis in plant breeding. Front Plant Sci. 5:389. doi:10.3389/fpls.2014.00389.

Hushiarian R, Yusof NA, Dutse SW. 2013. Detection and control of Ganoderma boninense: strategies and perspectives. SpringerPlus. 2(1):555. doi:10.1186/21931801-2-555.

Jaganathan D, Ramasamy K, Sellamuthu G, Jayabalan S, Venkataraman G. 2018. CRISPR for crop improvement: an update review. Front Plant Sci. 9:985. doi:10.3389/fpls.2018.00985.

Jinek M, Chylinski K, Fonfara I, Hauer M, Doudna JA, Charpentier E. 2012. A programmable dual-
RNA-guided DNA endonuclease in adaptive bacterial immunity. Science. 337(6096):816-821. doi:10.1126/science.1225829.

Mali P, Esvelt KM, Church GM. 2013. Cas9 as a versatile tool for engineering biology. Nat Methods. 10(10):957-963. doi:10.1038/nmeth.2649.

Ommelna BG, Jennifer AN, Chong KP. 2012. The potential of chitosan in suppressing Ganoderma boninense infection in oil-palm seedlings. J Sustain Sci Manag. 7(2):186-192.

Shen H, Strunks GD, Klemann BJPM, Hooykaas PJJ, de Pater S. 2017. CRISPR/Cas9-induced doublestrand break repair in Arabidopsis nonhomologous end-joining mutants. G3: Genes, Genomes, Genet. 7(1):193-202. doi:10.1534/g3.116.035204.

Tan YC, Yeoh KA, Wong MY, Ho CL. 2013. Expression profiles of putative defence-related proteins in oil palm (Elaeis guineensis) colonized by Ganoderma boninense. J Plant Physiol. 170(16):14551460. doi:10.1016/j.jplph.2013.05.009.

Telem RS, Wani HS, Singh NB, Nandini R, Sadhukhan R, Bhattacharya S, Mandal N. 2013. Cisgenics-a sustainable approach for crop improvement. Curr Genomics. 14(7):468-476. doi:10.2174/13892029113146660013.

Yang TH. 2007. Social Factors, Transaction Costs and Industrial Organization. Int Sociol. 22(4):435-461. doi:10.1177/0268580907078008. 